\title{
The Effects of Prosocial Cartoon Examples on Children's Donating Behavior
}

\author{
Qian Zhang' \\ ZheMin Duan' \\ Dan Xiang' \\ Yue Yu' \\ JingJin $\operatorname{Tian}^{2}$ \\ 'Center for Studies of Education and \\ Psychology of Minorities in Southwest \\ Area \& Faculty of Education, Chongqing, \\ People's Republic of China; ${ }^{2}$ Dawn \\ Innovation Kindergarten, Chongqing, \\ People's Republic of China
}

Purpose: In this study, we examined whether prosocial cartoons could inspire children to donate toys to others immediately upon exposure.

Participants and Methods: Cartoons were rated as prosocial or control via 80 adults. One hundred and fifty-six children participated in the study $\left(M_{\text {age }}=5.29, S D=0.79\right)$. Children in the experimental group were exposed to cartoons in which the main character had a large number of donating behaviors, while children in the control group watched cartoons without donating behaviors (randomized controlled study). They watched these cartoons for 4 consecutive days. Afterwards, children's donating behaviors toward their peers were assessed in the Toy Donation Task (TDT). An analytic method of 2 (cartoon: prosocial vs control) $\times 2$ (gender: male vs female) $\times 3$ (age: 4 vs 5 vs 6 ) analysis of variance (ANOVA) was used to make result analyses.

Results: The empirical results indicated that watching cartoons specifically depicting charitable donations (and not cartoons with other prosocial content) increased donations for charitable causes, whilst watching the control cartoons (cartoons without prosocial content) which did not depict characters acting in an antisocial way did not increase donating behavior. Specifically, 5-year-old female children reported more donating behavior than 6-year-old female children and 4-year-old female children, whilst no significant age effects were found among male children. Here, 4-6-year-old female children and 4-5-year-old male children in the prosocial cartoon condition reported more donating behavior than those in the control cartoon condition.

Conclusion: These findings indicated an accumulating positive effect of watching cartoons with donating content on children's donating behavior, especially for 4-6-year-old female children and 4-5-year-old male children.

Keywords: prosocial cartoons, donating behavior, toy donation task, children

\section{Introduction}

The relationship between prosocial media and children's donation behavior across cultures is an important topic. Although prior literature has emphasized the positive effects of prosocial cartoon viewing on prosociality outcomes, little experimental study has focused on the specific effects of prosocial cartoons giving as charitable donations on children's donating behavior in China. In other words, there is a relative lack of research on prosocial cartoons from Eastern cultures. For example, previous research has mainly focused on children's sharing, helping, cooperating and gratitude in Western nations, ${ }^{1,2}$ while the empirical research on the relationships between prosocial cartoon giving or donating as charitable donations and giving to organizations (donation behavior) was relatively sparse. Although we
Correspondence: Qian Zhang

Division of Early Childhood Education, Faculty of Education, Southwest

University, Chongqing, 4007I5, People's

Republic of China

Email zhq@swu.edu.cn 
believe that the study can make a valuable contribution to the knowledge and literature by analyzing the selected prosocial cartoon effects on young children's donation behavior, cultural variation issues should be considered since this study was conducted in China. Most importantly, different donation decision-making results may demonstrate the importance of cultural differences when trying to understand people's prosocial behavior. ${ }^{3}$ In view of this, it is necessary to evaluate the influence of the selected prosocial cartoon animations on children's donation behavior across cultures. This is the main reason why we should identify the gaps that need to be addressed to understand the state of the field in this body of knowledge.

Prosociality refers to any voluntary behavior aimed at benefiting others, and media can be an effective tool to understand the social preferences and motives behind such behavior. $^{4}$ Donating behavior is a powerful force of prosociality, which is measured through children's allocation of resources (eg, stickers) to needy peers. ${ }^{5}$ As such, donating behavior includes the distribution of goods to recipients in need (eg, children are asked if they are willing to donate candy to poor children). ${ }^{6}$ In a sense, donating behavior reflects the concern for disadvantaged groups, which is of great significance to children's prosocial development, ${ }^{7}$ so this is an important topic that needs to be further explored.

Prosocial cartoon is a type of cartoon that attempts to impart moral information (eg, Super Friends) in television programs, compared with control cartoon (eg, Bugs Bunny). ${ }^{8,9}$ However, prosocial behavior is a very broad concept including many behavioral aspects like helping, sharing, comforting, cooperating, etc. Notably, prosocial behavior mainly includes developmental patterns of donating and helping during infancy and early childhood. Drawing upon this, we choose the treatment cartoons to specifically depict charitable donations, while the control cartoons also have "positive" content (ie, they do not depict characters acting in an antisocial or selfish way). To avoid the label "prosocial cartoon" is too broadly formulated, we investigate whether watching cartoons specifically depicting donating for charitable causes (and not cartoons with other prosocial content) would increase subsequent donations for charitable causes. In the present study, we set up a specific donation situation for children to donate their bear dolls to their poor peers. The number of bear dolls represents the measure of donating behavior, ie, the more the number of bear dolls donated, the more donating behavior, and vice versa. General Learning
Model (GLM) details that prosocial-media use can increase children's prosocial thoughts and prosocial behavior. $^{11,12}$ Accordingly, we postulate that children may imitate donating cartoon examples (eg, cartoon models who try to donate their favorite items to others) to show donating behavior.

\section{Prosocial Media Exposure and Prosocial Behavior}

Previous studies have provided empirical evidence that exposure to prosocial video games can increase prosocial behaviors. ${ }^{13-16}$ More precisely, prosocial media increases donating and helping. ${ }^{17,18}$ Similarly, exposure to songs with prosocial lyrics increases the accessibility of prosocial thoughts, affect, and behavior. ${ }^{19}$ Notably, prosocial televised cartoon programs increase prosocial behavior in comparison to standard televised cartoon programs. ${ }^{15}$ Some researchers have also found that a prosocial televised example can increase children's helping and donating. $^{20,21}$ Why should prosocial media increase children's prosocial behavior and what are the underlying processes? Some researchers found prosocial media can increase the accessibility of prosocial thoughts, sympathy and empathy to cause prosocial behavior. ${ }^{14,16,18,22}$ Given that prosocial media exposure increases children's prosocial outcomes, donating cartoon scenes may increase children's donation behavior accordingly. Although there are multiple studies showing that consuming prosocial media increases prosocial behavior, the specific donating components of prosocial media sets our study apart from these previous studies. Given the large body of the literature on the connection between prosocial media exposure and prosocial behavior, the novelty value of the current study is that we expect that donating for charitable causes is fundamentally different from other aspects of prosocial behavior. Donating for charitable causes is particularly important for educators to cultivate Chinese children's donating behavior in educational practice.

\section{Gender and Prosocial Behavior}

Previous literature has indicated that females show more sympathy and prosocial behavior than males, and this gender difference increases with age. ${ }^{23,24}$ Likewise, women tend to exhibit more prosocial behavior than men. $^{25-27}$ There are three meta-analyses showing that women are significantly more altruistic than men in dictator games, and that promoting intuition relative to 
deliberation increases giving in a dictator game among women, but not among men. ${ }^{28-30}$ In addition, girls make significantly higher donations than boys of the same age. ${ }^{5}$ Thus, prosocial behaviors may be gender-specific, and it can be assumed that female children will show more donating behavior than male children after watching prosocial cartoons. It is of value to test the gender effects on children's donating behavior by conducting an experiment for an exploratory purpose.

\section{Age and Donating Behavior}

Empirical evidence indicated that age is related to donation behavior to some extent. For example, 6-year-olds display more donating behavior than 4-year-olds. ${ }^{31,32}$ Other research findings demonstrated that 8-year-olds donate significantly more than 4-year-olds. ${ }^{5}$ Interestingly, older children have a greater possibility of donating behavior than younger children because of higher donated motives. ${ }^{33}$ Nonetheless, children in Brazil, India and Canada are not willing to donate valuables to others with the increasing age. ${ }^{34}$ As such, there is a dispute over the age effects on children's donating behavior. Besides, previous research has shown that gender effects and age effects can be related to self-control as older children have higher self-control than younger children. ${ }^{35}$ Thus, it is necessary to test whether there are age effects on children's donating behavior after exposure to prosocial cartoons due to the lack of Chinese research. We also expect a different effect of exposure for children of different ages.

\section{The Present Experiment}

The objective of this study is to investigate the role of prosocial cartoons in the development of young children's donating behavior by conducting a $2 \times 2 \times 3$ factorial experiment. To this end, we analyze gender-specific and agespecific treatment effects with a special focus on two aspects of the experimental design, prosocial and control cartoons. Kindergartners are included because this is an age group that is largely unstudied concerning the effects of prosocial cartoons. ${ }^{36}$ Given that kindergartners face some interpersonal challenges, prosocial cartoon models may function as a source of inspiration to promote their social development. ${ }^{37}$ Based on the above, we propose the following hypotheses:

Hypothesis 1: Viewing prosocial cartoons will increase children's donating behavior in comparison to the control group.

Hypothesis 2: Female children will show more donating behavior than male children after viewing prosocial cartoons.

Hypothesis 3: Six-year olds will show more donating behavior than 5-year olds and 4-year olds after viewing prosocial cartoons.

\section{Methods}

\section{Samples and Procedure}

The study took place in Fall 2020. All procedures involving human participants in this study were conducted in accordance with the 1964 Helsinki Declaration. All procedures in this study were approved by the Ethics Committee at Southwest University in China. A total of 156 children aged from 4 to 6 years $\left(M_{\text {age }}=5.29\right.$, standard deviation $[S D]=0.79)$ were randomly selected from the Dawn and Innovation Kindergarten in Chongqing, Southwest of China. Based on the research literature that 8-year-olds report more donation behaviors than 4 -year-olds, ${ }^{5}$ we attempt to compare the difference in donating behavior among 4-year-olds, 5 -year-olds and 6-year-olds. It should be noted that we divided the age of children according to one year (full age), that is, the actual number of years of birth. This is a commonly used age calculation method in China's population statistics. Children were tested in a group setting. The research staff organized 12 groups, of which 13 children in each group were tested. These groups were structured as a whole sample. They were homogeneous regarding gender and age. In other words, one age group was composed of prosocial cartoon group and control cartoon group, so there were 6 groups in prosocial cartoon group (two gender groups in one age group) and 6 groups in control cartoon group, accounting for 12 groups in total (see Table 1). Participants

Table I Demographic Information of the Group Composition $(n=\mid 56)$

\begin{tabular}{|c|c|c|c|c|c|c|c|c|c|c|c|}
\hline \multicolumn{6}{|c|}{ Prosocial Cartoon Group } & \multicolumn{6}{|c|}{ Control Cartoon Group } \\
\hline \multicolumn{2}{|c|}{ 4-Year-Olds } & \multicolumn{2}{|c|}{ 5-Year-Olds } & \multicolumn{2}{|c|}{ 6-Year-Olds } & \multicolumn{2}{|c|}{ 4-Year-Olds } & \multicolumn{2}{|c|}{ 5-Year-Olds } & \multicolumn{2}{|c|}{ 6-Year-Olds } \\
\hline Male & Female & Male & Female & Male & Female & Male & Female & Male & Female & Male & Female \\
\hline 13 & 13 & 13 & 13 & 13 & 13 & 13 & 13 & 13 & 13 & 13 & 13 \\
\hline
\end{tabular}


watched assigned cartoons in a group setting (ie, one classroom as a unit) and completed the experiment in order. However, it was worth noting that 156 children were randomly recruited from 5 classrooms of the same kindergarten. There were 78 males and 78 females. There were 524 -yearolds, 52 5-year-olds, and 52 6-year-olds. To assure the consistency of donating behavior, none of the participants were found to be related to the charity based on an interview. None of them has experienced any form of charitable donations. Half of them were randomly assigned to watch prosocial cartoons depicting charitable donations, and the other half were randomly assigned to watch control cartoons. The children watched a 15-min assigned cartoon per day for 4 consecutive days. Then, their donating behaviors were tested.

The specific procedure was as follows: First, parents were told that their children were going to be assigned to watch 4 cartoons for 4 consecutive days (one cartoon per day), and then children's donation behavior would be assessed in a Toy Donation Task (TDT) or Bear Dolls Task (BDT). Parents were also told that their children could terminate the experiment at any time. After parents understood the experimental purposes and procedures, all parents gave informed consents to their children's participation, and the consent rates accounted for $100 \%$. However, parents were not at the place where children completed the TDT in the kindergarten. 156 children were randomly recruited from 5 classrooms in the same kindergarten. Second, children's pretest donation behaviors were individually measured in a TDT ( $0-5$ points). In other words, the TDT occurred for the first time. Third, half of children were randomly assigned to watch 4 prosocial cartoons with donation content or 4 control cartoons with no donation content (ie, each participant had the same opportunity) for 4 consecutive days, and each cartoon was watched for 15 minutes. Children watched these cartoons in a group setting. More precisely, all the children who watched assigned cartoons were in class under teacher supervision, and all the children watched cartoons at the same time in the same order. We wanted to examine the lasting effects of prosocial cartoons exposure in comparison with immediate effects, and thus we attempted to select 4 consecutive days. Actually, the whole study was conducted for 4 consecutive days in total, immediately after day 4 post-test happened. The pretest happened before children watched their assigned cartoons. The purpose of pretest was to test whether the base levels of donating behavior were homogeneous before the formal experiment. We must ensure that all participants in either the experimental or control groups yield no significant differences in donation behavior prior to the experiment. The pretest could assure that the outcomes be caused by the experimental variable (ie, prosocial cartoons). In other words, TDT was employed as the paradigm to measure children's donation behavior before or in the formal experiment. Notably, 13 participants were assessed for each condition (ie, each age, gender, and cartoon) with the help of lab assistants, and thus no missing data from pre- to post-test was found. Fourth, participants completed the TDT immediately after watching the fourth cartoon. Research assistants took children to a separate place when asking about their donating behaviors (0-5 points) in the absence of other students. Coding was done independently by 5 undergraduates who were not aware of the hypothesis or procedure. All the undergraduates are female. Because most undergraduates majoring in Child Psychology in our university are female students, the gender of our research assistants is female in this study. Finally, each participant received a nice gift as a reward.

\section{Materials}

Four prosocial cartoons (Peppa Pig Father's Donation by Running, Big Head Son and Little Head Father's Charity, Enthusiastic Kiki, Big Ear Tutu's Blood Donation) and four control cartoons (Peppa Pig Talent Day, Peppa Pig and Perfume, Peppa Pig Takes the Train, Peppa Pig Fishpond) were used as experimental materials. To exclude the confounding variables of cartoon contents, we specifically selected the different episodes of the same cartoon. Prosocial cartoons included many donation scenes and contents, whilst control cartoons did not include any donation contents and scenes. Peppa Pig Father's Donation by Running told that Peppa Pig's father collects money to fix the roof of Peppa Pig's school by running. Big Head Son and Little Head Father's Charity told that little head father shares the big head son with the difficulties of disadvantaged children in the mountain area, so the big head son donates his toys to them. Enthusiastic Kiki told that Kiki accidentally empties the waste products collected by Miaomiao. Kiki then collects plastic bottles, flyers and other waste products. Kiki and Miaomiao donate the money they get from selling waste products to disadvantaged children in mountain areas. Big Ear Tutu's Blood Donation told that Big Ear Tutu's father decides to donate blood, but his mother does not support his father because of his blood disease. His mother goes to donate blood and becomes a heroine. Peppa Pig Talent Day told 
that Peppa Pig plans to perform on talent day, but it is performed by someone else, so Peppa Pig finally steps in the mud. Peppa Pig Takes the Train told that kindergartens organize children to go out by train and finish their tasks. At the same time, kindergartners are taught to take good care of their own things. Peppa Pig and Perfume told that Peppa Pig and her brother George's story of making perfume in grandma's house. Peppa Pig Fishpond told that Peppa Pig's Father takes his family to the fishpond where he used to play.

Based on the media rating research literature, ${ }^{38}$ we chose prosocial cartoons and control cartoons by conducting a manipulation check. Eighty adults (60 undergraduates, 10 postgraduates, 10 kindergarten teachers; $M_{\text {age }}=20.54$, $S D=2.37$ ) were chosen to assess the donating attributes of the cartoons using a Likert 5-point scale ( 1 = very inconsistent to $5=$ very consistent). Ten adults assessed one cartoon, constituting for 8 groups. One-factor ANOVA was used to compare the donating attributes of cartoons in terms of the following rating criteria: (1) Donation Scenes (Does this cartoon have many donating scenes?), (2) Donation Contents (Does this cartoon have many donating contents?), (3) Interest (Is this cartoon interesting?), (4) Difficulty (Is this cartoon very difficult for kindergartners to understand?), (5) Action (Does this cartoon have many action plots?), (6) Pleasure (Are you pleased to watch this cartoon?). Peppa Pig Father's Donation by Running, Big Head Son and Little Head Father's Charity, Enthusiastic Kiki and Big Ear Tutu's Blood Donation got significant higher score than the other four cartoons in terms of Donation Scenes $[F(7,72)=35.36, p<0.001, d=1.35]$ and Donation Contents $[F(7,72)=30.69, p<0.001, d=$ 1.26]. However, there were no significant rating differences in Interest $[F(7,72)=1.68, p=0.13, d=0.29]$, Difficulty $[F$ $(7,72)=0.79, p=0.60, d=0.20]$, Action $[F(7,72)=1.37$, $p=0.23, d=0.27]$, and Pleasure $[F(7,72)=1.30, p=0.26$, $d=0.26]$. More specifically, we assumed the cartoon had the donating attribute if the mean score was higher than 3 points, while the cartoon did not have a donating attribute if the mean score was lower than 3 points. Thus, Peppa Pig Father's Donation by Running, Big Head Son and Little Head Father's Charity, Enthusiastic Kiki and Big Ear Tutu's Blood Donation were regarded as the cartoons with donation scenes and contents. Peppa Pig Talent Day, Peppa Pig and Perfume, Peppa pig Takes the Train and Peppa Pig Fishpond were regarded as the cartoons with no donation scenes and contents.

\section{Toy Donation Task}

To assess donating behavior, we provided 5 bear dolls as the toys that children would donate in a Toy Donation Task (TDT). Each bear doll was about $30 \mathrm{~cm}$ in length, $20 \mathrm{~cm}$ in width and $15 \mathrm{~cm}$ in height. All bear toys were identical, that is, they belonged to the same brand series. The reason why we chose bear doll (ie, Teddy Bear) as a donation toy was that it is a kind of neutral toy, which may reduce girls' gender preference for Barbie dolls and boys' gender preference for transformers and cars (potential confounders). In the donation situation created in this experiment, research staff specifically told the children that the bear toys you chose to donate to disadvantaged peers would be sent through the Donation Box.

To ensure the content validity of the bear dolls, we chose 10 kindergartners $\left(M_{\text {age }}=5.10, S D=0.84\right)$ to assess the 5 bear dolls in terms of (1) Likability (I like this bear doll very much) and (2) Interest (I am very interested in playing with this bear doll) from 1 point (very inconsistent) to 3 points (very consistent). The reason why we listed the responses including "inconsistent" (1 point), "uncertain" ( 2 points) and "consistent" (3 points) was to help children understand these items and test the statistical differences in Likability and Interest. One factor ANOVA revealed that there were no significant differences in Likability $[F(4,45)=0.79, p=$ $0.54, d=0.26]$ and Interest $[F(4,45)=0.77, p=0.55, d=$ 0.25 ] among these 5 bear dolls. Notably, the average score was higher than 2 points, indicating that children showed likability and interest for the bear dolls. The greater amount of donated bear dolls indicated more donating behavior of children, and vice versa. No donating behavior was available if the number of donated bear dolls was zero.

To date, various experimental paradigms were employed to measure children's donating behaviors. For example, researchers create emergency situations to induce children to donate to their peers in distress. ${ }^{39}$ In addition, experimenters should create donation situations to allow children to anonymously sacrifice their favorite valuables (eg, toys, money) to charity organizations and donate them to disadvantaged groups. ${ }^{40}$ In the current experiment, the research staff first took a group of 13 children to a specific room, organized them to watch different types of cartoons for 4 consecutive days, and then they completed the TDT. The research staff asked the participants,

Hey kids, thank you for watching the cartoons. We give you 5 bear dolls as a reward. Do you like them? However, there are many poor/disadvantaged children who never have their 
own bear dolls. They like bear dolls very much. Would you like to donate your bear dolls to them, please? If so, how many are you going to donate (0-5 points)? The bear dolls will be sent to disadvantaged peers via China Hope Project.

The children took the bears back with them if they were not willing to donate (ie, no donation behavior was also available). All of the children were told so in the beginning. The children were questioned individually, and each was questioned consecutively. They put their bear dolls they were willing to donate in the Donation Box. The research staff recorded the number of bear dolls donated by each participant as a measure of donation behavior.

\section{Validation of the Materials}

In the present study, we have chosen 80 adults to rate the cartoon materials (ie, 4 prosocial vs 4 control) and 10 kindergartners to rate the toy materials (ie, 5 bear dolls) to ensure the good validation of the materials. Overall, we selected 4 prosocial cartoons and 4 control cartoons in terms of Donation Scenes $[F(7,72)=35.36, p<0.001, d=1.35]$ and Donation Contents $[F(7,72)=30.69, p<0.001, d=1.26]$. In addition, we selected the 5 bear dolls as donating materials in terms of high (Average score $>2$ points) but not significant differences in Likability $[F(4,45)=0.79, p=0.54, d=0.26 ; M=2.22$, $S D=0.76]$ and Interest $[F(4,45)=0.77, p=0.55, d=0.25$; $M=2.12, S D=0.77]$. Thus, the validation of the cartoon materials and toy materials was guaranteed.

\section{Design}

A 2 (cartoon: prosocial vs control) x 2 (gender: male vs female) x 3 (age: 4 vs 5 vs 6) experimental-control design was conducted. The independent variables were cartoon, gender, and age. The dependent variable was donating behavior (the number of bear dolls children donated to disadvantaged peers).

\section{Results}

\section{The Pretest Levels of Donating Behavior}

Independent sample $t$-tests were performed to test whether there were significant differences in donation behavior between prosocial cartoon group and control cartoon group, as well as male children group and female children group. Meanwhile, a one-factor analysis of variance (ANOVA) was conducted to test whether there were significant differences in donation behavior among 4-yearolds, 5-year-olds, and 6-year-olds.

Table 2 shows the pretest data for donating behavior. We used the data from the pretest to establish that there was no difference in donation propensity. Overall, the pretest level of donating behavior was not significant between the experimental group and the control group $[t(154)=-1.24, p=$ $0.22, d=-0.20]$. Similarly, the pretest level of donating behavior was not significant between male group and female group $[t(154)=0.53, p=0.60, d=0.10]$. The pretest level of donating behavior was not significant among 4-year-olds, 5-year-olds, and 6-year-olds $[F(2$, $153)=1.01, p=0.37, d=0.16]$. As such, participants in both groups demonstrated the relatively homogeneous level of donating behavior prior to the experiment.

\section{Descriptive Statistics}

Table 3 shows the means and standard deviations of donating behavior by gender and age in the present study.

\section{Analysis of Variance on Donating Behavior}

A 2 (cartoon) x 2 (gender) x 3 (age) ANOVA was done to test hypotheses 1-3 (Table 4). Cartoon, gender and age were independent variables, while donating behavior was dependent variable. A simple effect analysis would be further carried out if the interaction between the independent variables had a significant impact on the dependent variables. The main effect of cartoon on donating behavior was significant. Children who watched prosocial cartoons showed more donating behavior than those who watched control cartoons. As a result, prosocial cartoon scenes emerged as a significant predictor of donating behavior. The main effects of gender and age on donating behavior were not significant. The gender $\mathrm{x}$ age interaction in donating behavior was significant. A simple effect analysis

Table 2 Pretest Data for Donating Behavior

\begin{tabular}{|c|c|c|c|c|c|c|c|}
\hline Outcomes & 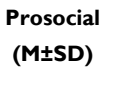 & 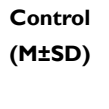 & $\begin{array}{c}\text { Male } \\
(\mathrm{M} \pm \mathrm{SD})\end{array}$ & $\begin{array}{l}\text { Female } \\
(\mathrm{M} \pm \mathrm{SD})\end{array}$ & $\begin{array}{c}\text { 4-Year-Olds } \\
\text { (M士SD) }\end{array}$ & 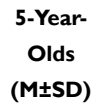 & 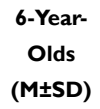 \\
\hline Donation & $2.29 \pm 1.30$ & $2.56 \pm 1.41$ & $2.49 \pm 1.35$ & $2.37 \pm 1.38$ & $2.21 \pm 1.42$ & $2.56 \pm 1.33$ & $2.52 \pm 1.32$ \\
\hline
\end{tabular}


Table 3 Means and Standard Deviation of Donating Behavior by Gender and Age

\begin{tabular}{|c|c|c|c|c|c|c|c|c|}
\hline \multirow[t]{2}{*}{ Age } & \multicolumn{2}{|c|}{ Prosocial Cartoons } & \multirow[t]{2}{*}{$\mathbf{N}$} & \multicolumn{2}{|c|}{ Control Cartoons } & \multirow[t]{2}{*}{$\mathbf{N}$} & \multirow[t]{2}{*}{ Total (M $\pm S D)$} & \multirow[t]{2}{*}{$\mathbf{N}$} \\
\hline & Male (M $\pm S D)$ & Female (M $\pm S D)$ & & Male (MISD) & Female (M $\pm S D)$ & & & \\
\hline 4-year-olds & $4.23 \pm 0.73$ & $3.92 \pm 1.04$ & 26 & $3.38 \pm 0.77$ & $2.62 \pm 1.33$ & 26 & $3.54 \pm 1.15$ & 52 \\
\hline 5-year-olds & $4.38 \pm 0.77$ & $4.62 \pm 0.51$ & 26 & $2.85 \pm 1.57$ & $3.62 \pm 1.19$ & 26 & $3.87 \pm 1.27$ & 52 \\
\hline 6-year-olds & $4.23 \pm 1.01$ & $4.62 \pm 0.51$ & 26 & $3.46 \pm 1.66$ & $2.15 \pm 0.90$ & 26 & $3.62 \pm 1.43$ & 52 \\
\hline Total & $4.28 \pm 0.83$ & $4.38 \pm 0.78$ & 78 & $3.23 \pm 1.39$ & $2.79 \pm 1.28$ & 78 & $3.67 \pm 1.29$ & 156 \\
\hline
\end{tabular}

Table 4 Analysis of Variance on Donating Behavior

\begin{tabular}{|c|c|c|c|}
\hline Variables & Mean Square & $F(d f 1, d f 2)$ & $\eta_{p}^{2}$ \\
\hline Cartoon & 68.01 & $60.22 * * *(1,144)$ & 0.28 \\
\hline Gender & 1.08 & $0.96(1,144)$ & 0.006 \\
\hline Age & 1.52 & $1.35(2,144)$ & 0.009 \\
\hline Cartoon $\times$ Gender & 2.83 & $2.50(1,144)$ & 0.02 \\
\hline Cartoon $\times$ Age & 0.97 & $0.86(2,144)$ & 0.006 \\
\hline Gender $\times$ Age & 4.35 & $3.85 *(2,144)$ & 0.02 \\
\hline Cartoon $\times$ Gender $\times$ Age & 4.06 & $3.59 *(2,144)$ & 0.02 \\
\hline
\end{tabular}

Notes: $* * * p<0.001, * p<0.05$

indicated that 5-year-old female children showed more donating behavior than 6-year-old and 4-year-old female children, whilst no significant age effects in donating behavior were found among male children (Table 5 and Figure 1). The cartoon $\mathrm{x}$ gender $\mathrm{x}$ age interaction on donating behavior was small but significant. A simple effect analysis indicated that 4-year-old male children in prosocial cartoon condition showed more donating behavior than 4-year-old male children in control cartoon condition. Four-year-old female children in prosocial cartoon condition showed more donating behavior than 4-year-old female children in control cartoon condition. Five-year-old male children in prosocial cartoon condition showed more donating behavior than 5-year-old male children in control cartoon condition. Five-year-old female children in prosocial cartoon condition showed more donating behavior than 5-year-old female children in control cartoon condition. Six-year-old female children in prosocial cartoon condition showed more donating behavior than 6-yearold female children in control cartoon condition.
However, no significant group differences in donating behavior were found in 6-year-old male children (Table 6, Figures 2 and 3). The cartoon $\mathrm{x}$ gender and cartoon $\mathrm{x}$ age interactions on donating behavior were not significant.

\section{Discussion}

In general, findings of the study highlighted the importance of using prosocial cartoon examples/models, which was an effective way to change children's donating behavior in the short term. Our study advanced the existing literature by giving the following strong points: First, despite general prosocial media (ie, video games) promoting donation, little empirical research touches on the impact of donation-themed cartoons on donating behavior, especially among Chinese kindergartners. Since kindergarten children were most likely to watch cartoons in their pastime, research on the relationship between donationthemed cartoons and donation behavior was much-needed. This was the primary reason why the need for donationthemed cartoons in our experimental study. Second, the study conducted in Eastern cultures could further replicate the finding that prosocial media exposure increased prosocial behavior among adolescents in Western cultures. As such, prosocial cartoon examples might also be rendered as ideal models to foster donating behavior for young Chinese children. The implications culture-wise gave us an insight into the effects of prosocial cartoons on donation in individualistic-oriented Western culture that could also apply to kindergartners in collectivist-oriented Chinese culture. Third, this research could provide useful information for the development of prosocial behavior by

Table 5 The Gender x Age Interaction on Donating Behavior

\begin{tabular}{|c|c|c|c|c|c|}
\hline Gender & 4-Year-Olds (M $\pm S E)$ & 5-Year-Olds (M $\pm S E)$ & 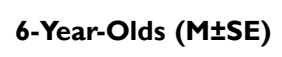 & $F(d f l, d f 2)$ & $\eta_{p}^{2}$ \\
\hline Male & $3.8 I \pm 0.21$ & $3.62 \pm 0.21$ & $3.85 \pm 0.21$ & $0.35(2,144)$ & 0.002 \\
\hline Female & $3.27 \pm 0.21$ & $4.12 \pm 0.21$ & $3.39 \pm 0.21$ & $4.85 * *(2,144)$ & 0.03 \\
\hline
\end{tabular}

Note: $* * p<0.01$. 


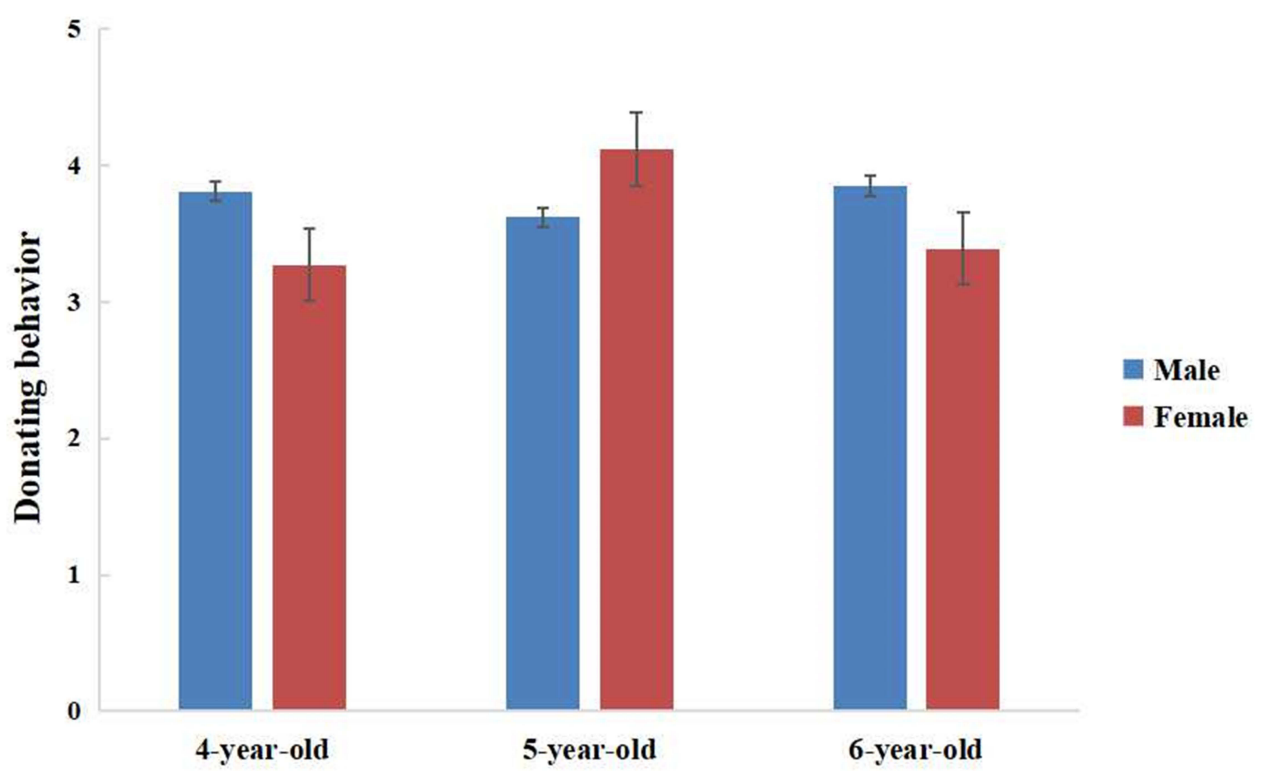

Figure I Gender x age interaction on children's donating behavior.

selecting fruitful donation-themed cartoons. Previous researchers have explored the effective measures to increase children's prosocial behavior, and our research might indicate that prosocial cartoon scenes can promote the development of donating behavior in Chinese kindergartners.

Consistent with Hypothesis 1, we found that children exposed to prosocial cartoons showed more donating behavior than those exposed to control cartoons after 4 consecutive days. The finding indicated an accumulating positive effect, which was consistent with the GLM that prosocial media could change individual's cognition, attitude, affection and behavior. ${ }^{11}$ Also, the finding confirmed that children could automatically mimic prosocial behavior from media role models. ${ }^{41}$ The finding replicated previous literature that prosocial media setting could immediately increase children's donation rates, donor prosociality, helping behavior, and altruistic behavior. ${ }^{42-44}$ Children in prosocial cartoon condition showed more donating behavior than those in control cartoon condition. The explanation of the results was that prosocial cartoon scenes might provoke more altruistic cognitive networks of children than control cartoons. In view of this, cartoon developers, parents and teachers might use prosocial cartoons with donated contents to develop children's donating behavior in real-world settings.

Inconsistent with Hypothesis 2, no significant gender effects on donating behavior were found in both prosocial cartoon group and control cartoon group. This finding also aligned with previous study that there were no significant gender main effects and media $\mathrm{x}$ gender interaction on prosocial behavior. ${ }^{45}$ Likewise, according to another study, there were no significant gender effects. ${ }^{35}$ However, the average level of donating behavior among female children was slightly higher than that of male children after viewing prosocial cartoons, though not statistically significant. Note that one possible explanation for the non-significant differences in donating behavior between male and female children was that gender differences in donations appear later in the development. ${ }^{46,47}$

Table 6 The Cartoon $\times$ Gender $\times$ Age Interaction on Donating Behavior

\begin{tabular}{|c|c|c|c|c|c|c|c|c|}
\hline \multirow[t]{2}{*}{ Age } & \multicolumn{4}{|c|}{ Male } & \multicolumn{4}{|c|}{ Female } \\
\hline & Prosocial (M $\pm S E)$ & Control (M \pm SE) & $F(d f l, d f 2)$ & $\eta_{p}^{2}$ & Prosocial (M \pm SE) & Control $(\mathrm{M} \pm \mathrm{SE})$ & $F(d f 1, d f 2)$ & $\eta_{p}^{2}$ \\
\hline 4-year-olds & $4.23 \pm 0.30$ & $3.39 \pm 0.30$ & $4.12 *(1,144)$ & 0.03 & $3.92 \pm 0.30$ & $2.62 \pm 0.30$ & $9.84^{* *}(1,144)$ & 0.06 \\
\hline 5-year-olds & $4.39 \pm 0.30$ & $2.85 \pm 0.30$ & $13.62^{* * *}(1,144)$ & 0.08 & $4.62 \pm 0.30$ & $3.62 \pm 0.30$ & $5.76 *(1,144)$ & 0.04 \\
\hline 6-year-olds & $4.23 \pm 0.30$ & $3.46 \pm 0.30$ & $3.41(1,144)$ & 0.02 & $4.62 \pm 0.30$ & $2.15 \pm 0.30$ & $34.88^{* * *}(1, \mid 44)$ & 0.18 \\
\hline
\end{tabular}

Notes: $* * * p<0.001, *_{p}<0.01, *_{p}<0.05$. 


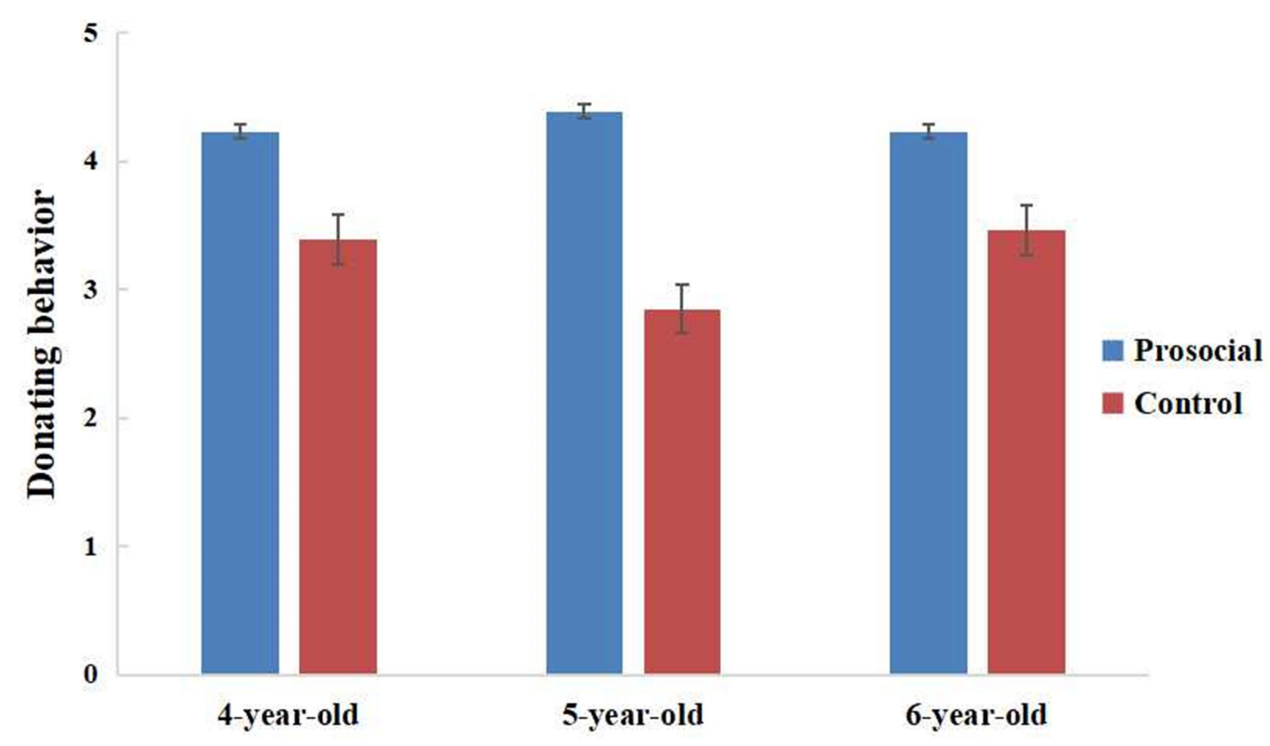

Figure 2 Cartoon $x$ gender $x$ age interaction on donating behavior among male children.

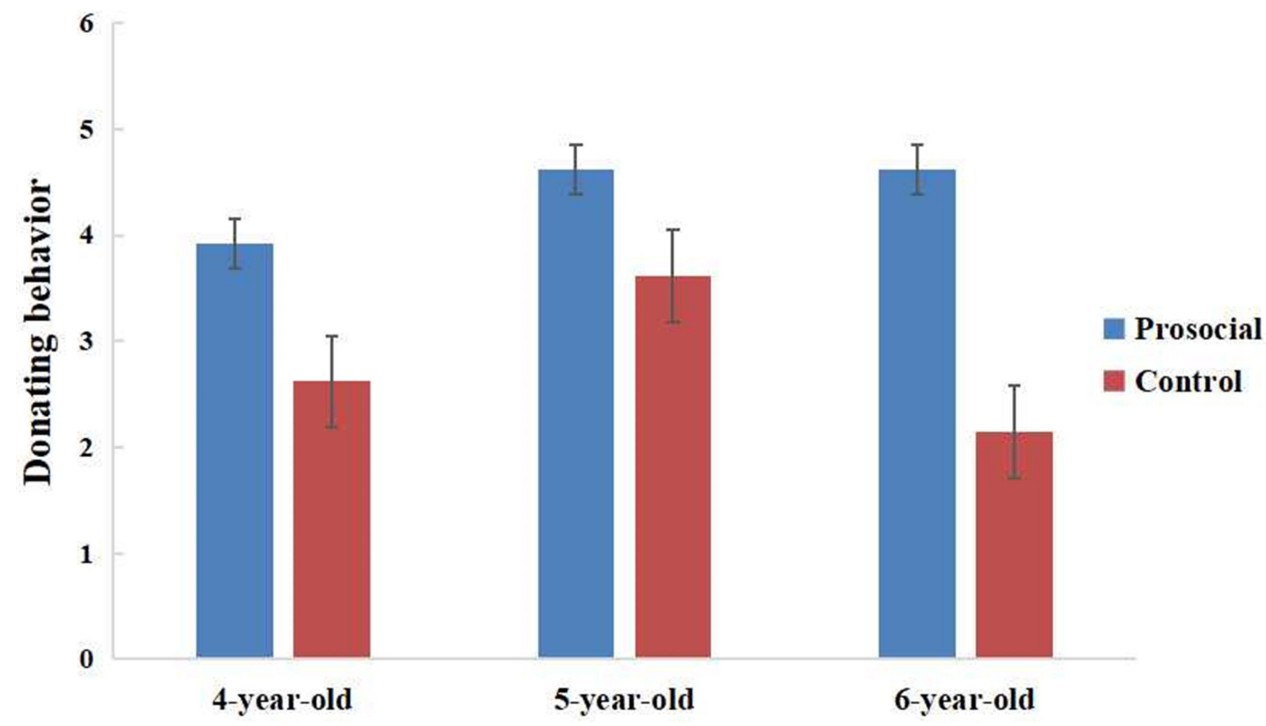

Figure 3 Cartoon $\mathrm{x}$ gender $\mathrm{x}$ age interaction on donating behavior among female children.

This might explain the results regarding non-significant gender differences in donating behavior.

Inconsistent with Hypothesis 3 of age effects, we found that 5-year-old female children demonstrated more donating behavior than 6-year-old female children and 4-year-old female children, whilst no significant age effects were found in male children. The finding was somewhat different from previous research showing that older children had more donating behaviors than younger children. ${ }^{5}$ In addition, the cartoon $\mathrm{x}$ gender $\mathrm{x}$ age interaction on donating behavior was significant. Specifically, female children aged 4 to 6 in prosocial cartoon condition showed more donating behaviors than those in control cartoon condition, whilst no significant group differences in donating behaviors were found among six-year-old male children. Perhaps educators should use prosocial cartoons to foster donation behavior among female children aged 4 to 6 and male children aged 4 to 5 . The finding suggested that these children might be the key groups for donating behavior development under the condition of prosocial cartoon scenes.

\section{Implications of the Findings}

The conclusions of this study provide a useful reference for the cultivation of children's donating behavior. First of 
all, perhaps increasing the exposure to prosocial cartoon scenes is an effective way to foster children's donating behavior. However, it is worth noting that 4 consecutive days of watching cartoons can only impact donation or altruistic behavior in the short run from a pedagogical perspective. Watching cartoons needs to be combined with other activities that foster altruism in the long run. In other words, watching cartoons only 4 days cannot make these kids altruists forever and this finding should be cautiously generalized to the real world. Secondly, given that 5-year-old female children show more donating behavior than 6-year-old female children and 4-year-old female children, educators should pay more attention to the cultivation of donating behaviors of 6-year-old female children and 4-year-old female children. Thirdly, given that prosocial cartoon scenes cause more donating behaviors in 4-6-year-old female children and 4-5-year-old male children than the control cartoon scenes, educators should shed light on the cultivation of donating behavior of 4-6-year-old female children and 4-5-year-old male children. Finally, given that no significant gender effects were found in donating behavior, educators may not care too much about focusing on the cultivation of donating behavior for male or female children.

\section{Limitations and Future Research}

This study has some limitations. First, although children watch assigned cartoons for 4 consecutive days, this experiment is not a real longitudinally manipulated one. As a result, it may be difficult to assess whether the limited time period of impact (ie, 15 minutes of daily exposure) will be more than short term. Future research should consider collecting longitudinal experimental data to determine the causal effects of prosocial cartoon scenes on children's donating behavior. Second, we did not include age as a continuous factor in the statistical analysis. Indeed, taking age as a categorical variable may lose some statistical power to some extent. Future studies need to consider age as a continuous factor in data analysis. Third, the way donation task administered was not only measuring the altruism of children but also measuring obedience of children. Ideally, we should give the children at least not to donate and do not ask them to donate, but only give them the options to donate. However, we asked them whether or how many they would like to donate may bias the results as children have tendency to do what teachers told them to do which may be obedience rather than prosociality to some extent. Future research should ideally provide them the options to donate rather than simply asking them to donate to improve the validity of donation measurement. Finally, the results of this study should be interpreted with caution in psychology research and donating behavior management practice due to the small sample size. In addition, the 5 bear dolls may not be children's favorite toys in reality, although they were rated as having with high Likability and Interest in our study.

\section{Strengths}

Despite such limitations, our study has several strengths and provides a unique contribution to a better understanding of the effects of selected prosocial cartoons on donating behavior. First, we selected four prosocial cartoons and four control cartoons based on rated donating contents and scenes. Second, the unique findings of our study were consistent with prior relevant results in a new cultural setting, and the study results supported the GLM that repeated exposure to prosocial cartoons increases children's donating behaviors. Third, the experimental design, random selection of children and the careful controls at various stages of the procedures allowed us to draw causal inferences about the prosocial cartoon effects on donating behavior. In particular, we evaluated the positive effects of prosocial cartoons sub-themes (ie, charitable donation) on donating behavior, which surpassed previous research that only tested the overall impact of prosocial media on prosocial behavior. Finally, it was worth noting that we measured the pretest level of children's donating behavior to ensure that they were homogeneous in both groups. The pretests conducted individually before the experiment could provide stronger conclusions than extant research.

\section{Conclusion}

This was an experimental study designed to test the effects of a series of cartoons featuring donation behavior on children's donating behavior, assessed by a TDT (or Bear Doll Task). We found empirical support for the efficacy of these cartoons with an immediate post-test. Our work specifically focused on donation, which was an important aspect of prosocial development. The study could contribute to the prosocial media literature and knowledge by analyzing the influence of the selected prosocial cartoons on children's donating behavior. 


\section{Data Sharing Statement}

Data supporting the findings presented in the current study will be available from the following places: https://osf.io/ae78r/.

\section{Ethical Statement}

Our study involved human experiments. Meanwhile, the current paper did not involve any sensitive topics that may make the participants feel uncomfortable about deception or concealment of participation. Based on the above reasons, the research is deemed to meet the institutional requirements and is therefore exempt from ethical recognition. All participants were informed of the research process and provided written informed consent in accordance with the Declaration of Helsinki. The principals of the kindergarten whose children were enrolled in this study have approved our experiment. The above study procedure has been approved by the Institutional Review Committee of the Faculty of Education, Southwest University.

\section{Acknowledgment}

This paper was supported by the National Social Science Foundation of China (17CSH006), the Chongqing Talent Plan Project (2021), the Central University's Fundamental Scientific Grant (SWU2009201), the QingMa Grant of Social Sciences in Chongqing (2020QM06), the Grant of Planned Social Sciences in Chongqing (2017YBJY085), and the Grant of Basic Education Quality Monitoring Collaborative Innovation Center in China (2020-06-005BZPK01). We would like to thank Ms. JingJin Tian, head of Dawn Innovation Kindergarden, for her organization and arrangement, as well as all kindergarten children participating in this study.

\section{Disclosure}

The authors report no conflicts of interest in this work.

\section{References}

1. Ulber J, Hamann K, Tomasello M. Extrinsic rewards diminish costly sharing in 3-year-olds. Child Dev. 2016;87(4):1192-1203. doi:10.1111/ cdev. 12534

2. Weller D, Lagattuta KH. Helping the in-group feels better: children's judgments and emotion attributions in response to prosocial dilemmas. Child Dev. 2012;84(1):253-268. doi:10.1111/j.1467-8624.2012.01837.x

3. Wang Y, Tang Y-Y, Wang J, Ma Y. Cultural differences in donation decision-making. PLoS One. 2015;10(9):e0138219. doi:10.1371/journal.pone. 0138219

4. Fehr E, Fischbacher U. The nature of human altruism. Nature. 2003;425(10):785-791. doi:10.1038/nature02043
5. Ongley SF, Nola M, Malti T. Children's giving: moral reasoning and moral emotions in the development of donation behaviors. Front Psychol. 2014;12(5):458-466. doi:10.3389/fpsyg.2014.00458

6. Rubin KH, Schneider FW. The relationship between moral judgment, egocentrism, and altruistic behavior. Child Dev. 1973;44(3):661-665. doi: $10.2307 / 1128027$

7. Hao J. Do children with better inhibitory control donate more? Differentiating between early and middle childhood and cool and hot inhibitory control. Front Psychol. 2017;8(12):21-29. doi:10.3389/fpsyg.2017.02182

8. Liss MB, Reinhardt LC. Aggression on prosocial television programs. Psychol Rep. 1980;46(3):1065-1066. doi:10.2466/pr0.1980.46.3c.1065

9. Meng Q, Sheng X, Zhao J, Wang Y, Su Z. Influence of mothers/grandmothers coviewing cartoons with children on children's viewing experience. Front Psychol. 2020;11(6):1232-1242. doi:10.3389/ fpsyg.2020.01232

11. Buckley KE, Anderson CA. A theoretical model of the effects and consequences of playing video games. In: Vorderer P, Bryant J, editors. Playing Video Games-Motives, Responses, and Consequences. LEA; 2006:363-378.

12. Gentile DA, Groves C, Gentile JR. The general learning model: unveiling the learning potential from video games. In: Blumberg FC, editor. Learning by Playing: Video Gaming in Education. Oxford University Press; 2014:121-142. doi:10.1093/ acprof:osobl/9780199896646.003.0009

13. Gentile DA, Anderson CA, Yukawa S, et al. The effects of prosocial video games on prosocial behaviors: international evidence from correlational, longitudinal, and experimental studies. Pers Soc Psychol Bull. 2009;35(6):752-763. doi:10.1177/0146167209333045

14. Greitemeyer T, Osswald S. Playing prosocial video games increases the accessibility of prosocial thoughts. J Soc Psychol. 2011;151 (2):121-128. doi:10.1080/00224540903365588

15. Greitemeyer T, Osswald S. Effects of pro-social video games on pro-social behavior. J Pers Soc Psychol. 2010;98(2):211-221. doi:10.1016/j.jesp.2009.04.005

16. Prot S, Gentile DA, Anderson CA, et al. Long-term relations among prosocial-media use, empathy, and prosocial behavior. Psychol Sci. 2014;25(2):358-368. doi:10.1177/0956797613503854

17. Chambers JH, Ascione FR. The effects of prosocial and aggressive video games on children's donating and helping. J Genet Psychol. 1987;148(4):499-505. doi:10.1080/00221325.1987.10532488

18. Greitemeyer T. Effects of prosocial media on social behavior: when and why does media exposure affect helping and aggression? Curr Dir Psychol Sci. 2011;20(4):251-255. doi:10.1177/0963721411415229

19. Greitemeyer T. Effects of songs with prosocial lyrics on prosocial thoughts, affect, and behavior. J Exp Soc Psychol. 2009;45 (1):186-190. doi:10.1016/j.jesp.2008.08.003

20. Guo R, He ZB, Wu Z. Emotion makes a difference: induced sadness reduces preschool boys' sharing behavior. Evol Hum Behav. 2019;40 (2):148-155. doi:10.1016/j.evolhumbehav.2018.10.001

21. Sprafkin JN, Liebert RM, Poulos RW. Effects of a prosocial televised example on children's helping. J Exp Child Psychol. 1975;20 (1):119-126. doi:10.1016/0022-0965(75)90031-4

22. Eisenberg N, Fabes RA, Spinrad TL. Prosocial behavior. In: Eisenberg N, Damon W, Lerner RM, editors. Handbook of Child Psychology (Vol. 3), Social, Emotional, and Personality Development. 6th ed. New York, NY: John Wiley \& Sons; 2006:646-718.

23. Eisenberg N, Fabes RA. Prosocial development. In: Damon W, Eisenberg N, editors. Handbook of Child Psychology: Social, Emotional, and Personality Development. Vol. 3. 5th ed. New York: John Wiley \& Sons; 1998:701-778.

24. Eisenberg N, Fabes RA, Schaller M, Miller PA. Sympathy and personal distress: development, gender differences, and interrelations of indexes. New Dir Child Adolesc Dev. 2010;1989(44):107-126. doi:10.1002/cd.23219894408 
25. Kuhnert R, Begeer S, Fink E, Rosnay MD. Gender-differentiated effects of theory of mind, emotion understanding, and social preference on prosocial behavior development: a longitudinal study. $J \operatorname{Exp}$ Child Psychol. 2017;154(2):13-27. doi:10.1016/j.jecp.2016.10.001

26. Espinosa MP, Kováŕík J. Prosocial behavior and gender. Front Behav Neurosci. 2015;14(4):88-97. doi:10.3389/fnbeh.2015.00088

27. Rand DG. Social dilemma cooperation (unlike dictator game giving) is intuitive for men as well as women. J Exp Soc Psychol. 2017;73 (11):164-168. doi:10.1016/j.jesp.2017.06.013

28. Brañas-Garza P, Capraro V, Rascon-Ramirez E. Gender differences in altruism on mechanical turk: expectations and actual behaviour. Econ Lett. 2018;170(9):19-23. doi:10.1016/j.econlet.2018.05.022

29. Engel C. Dictator games: a meta study. Exp Econ. 2011;14 (4):583-610. doi:10.1007/s10683-011-9283-7

30. Rand DG, Brescoll VL, Everett JAC, Capraro V, Barcelo H. Social heuristics and social roles: intuition favors altruism for women but not for men. J Exp Psychol Gen. 2016;145(4):389-396. doi:10.1037/ xge 0000154

31. Ji CL, Gao LL. The relationship between role taking and sharing among 3-6-old children. Chin J Health Psychol. 2017;25(1):126. doi: CNKI:SUN:JKXL.0.2017-01-030

32. Li D. The relationship between children's role taking ability and altruistic behavior development. Chin J Psychol Dev Educ. 1994;2:8-10, 14. doi:CNKI:SUN:XLFZ.0.1994-02-001

33. Raviv A, Bar-Tal D, Lewis-Levin T. Motivations for donation behavior by boys of three different ages. Child Dev. 2016;51(2):610-613. doi: $10.2307 / 1129306$

34. Corbita J, Callaghana T, Svetlovab M. Toddlers' costly helping in three societies. J Exp Child Psychol. 2020;195:104841. doi:10.1016/j. jecp.2020.104841

35. Ugur ZB. Does self-control foster generosity? Evidence from ego depleted children. J Behav Exp Econ. 2021;90(1):101652. doi:10.1016/j.socec.2020.101652

36. Strasburger VC. Children, adolescents and the media: what we know, what we don't know and what we need to find out (quickly!). Arch Dis Child. 2009;94(9):655-657. doi:10.1136/adc.2008.157156

37. Zhang Q, Cao Y, Tian JJ, Kim EL, Gentile DA. Effects of prosocial cartoon models on aggressive cognitions and aggressive behaviors. Child Youth Serv Rev. 2020;118(11):105498. doi:10.1016/j. childyouth.2020.105498
38. Adachi PJC, Willoughby T. The effect of violent video games on aggression: is it more than just the violence? Aggress Violent Behav. 2011;16(1):55-62. doi:10.1016/j.avb.2010.12.002

39. Marshall J, Wynn K, Bloom P. Do children and adults take social relationship into account when evaluating people's actions? Child Dev. 2020;91(5):e1082-e1100. doi:10.1111/cdev.13390

40. Karafantis DM, Levy SR. The role of children's lay theories about the malleability of human attributes in beliefs about and volunteering for disadvantaged groups. Child Dev. 2004;75(1):236-250. doi:10.1111/j.1467-8624.2004.00666.x

41. Bandura A. Social cognitive theory for personal and social change by enabling media. In: Singhal A, Cody MJ, Rogers EM, Sabido M, editors. LEA's Communication Series. Entertainment-Education and Social Change: History, Research, and Practice. Lawrence Erlbaum Associates Publishers; 2004:75-96.

42. Gelfand DM, Hartmann DP, Cromer CC, Smith CL, Page BC. The effects of instructional prompts and praise on children's donation rates. Child Dev. 1975;46(4):980-983. doi:10.2307/1128408

43. Mcguigan N, Fisher R, Glasgow R. The influence of receiver status on donor prosociality in 6- to 11-year-old children. Child Dev. 2016;87(3):855-869. doi:10.1111/cdev.12517

44. Rebecca NHDL, Christa AVL. Helping behavior in Disney animated movies and children's helping behavior in the Netherlands. J Child Media. 2018;12(2):159-174. doi:10.1080/17482798.2017.1409245

45. Afolabi OA, Idowu EO. Influence of gender, spiritual involvement/ belief and emotional stability on prosocial behavior among some Nigerian drivers. Can Social Sci. 2014;10(1):121-127. doi:10.3968/ j.css.1923669720141001.3919

46. Capraro V. Gender differences in the trade-off between objective equality and efficiency. Judgm Decis Mak. 2020;15(4):534-544.

47. Messer EJE, Burgess V, Sinclair M, Grant S, Spencer D, Mcguigan N. Young children display an increase in prosocial donating in response to an upwards shift in generosity by a same-aged peer. Sci Rep. 2017;7(1):2633-2645. doi:10.1038/ s41598-017-02858-y
Psychology Research and Behavior Management

\section{Publish your work in this journal}

Psychology Research and Behavior Management is an international, peer-reviewed, open access journal focusing on the science of psychology and its application in behavior management to develop improved outcomes in the clinical, educational, sports and business arenas. Specific topics covered in the journal include: Neuroscience, memory and decision making; Behavior modification and management; Clinical

\section{Dovepress}

applications; Business and sports performance management; Social and developmental studies; Animal studies. The manuscript management system is completely online and includes a very quick and fair peer-review system, which is all easy to use. Visit http://www. dovepress.com/testimonials.php to read real quotes from published authors. 